\title{
Structuring from nanoparticles in oil-based ferrofluids
}

\author{
Z. Rozynek ${ }^{1, a}$, A. Józefczak ${ }^{2}$, K.D. Knudsen ${ }^{3}$, A. Skumiel ${ }^{2}$, T. Hornowski ${ }^{2}$, J.O. Fossum¹ ${ }^{1}$ M. Timko ${ }^{4}$, P. Kopčanský4 ${ }^{4}$ \\ and M. Koneracká ${ }^{4}$ \\ 1 Department of Physics, NTNU, Høgskoleringen 5, NO-7491 Trondheim, Norway \\ 2 Institute of Acoustics, Faculty of Physics, UAM, Umultowska 85, 61-614 Poznań, Poland \\ 3 Physics Department, IFE, 2027 Kjeller, Norway \\ 4 Institute of Experimental Physics, SAV, Watsonova 47, 04001 Košice, Slovakia
}

Received 10 October 2010 and Received in final form 1 February 2011

Published online: 18 March 2011

(c) The Author(s) 2011. This article is published with open access at Springerlink.com

\begin{abstract}
The effect of magnetic field on the structure formation in an oil-based magnetic fluid with various concentrations of magnetite particles was studied. The evaluation of the experimental data obtained from small-angle X-ray scattering and ultrasonic attenuation indicates the formation of chain-like aggregates composed of magnetite particles. The experimental data obtained from ultrasonic spectroscopy fit well with the recent theoretical model by Shliomis, Mond and Morozov but only for a diluted magnetic fluid. In this model it is assumed that a dimer is the main building block of a $B$-field-induced chain-like structure, thus the estimation of the nematic order parameter does not depend on the actual length of the structure. The scattering method used reveals information about the aggregated structure size and relative changes in the degree of anisotropy in qualitative terms. The coupling constant $\lambda$, concentrations $\phi$, average particle size $d$ and its polydispersity $\sigma$ were initially obtained using the vibrating sample magnetometry and these results were further confirmed by rheometry and scattering methods. Both the particles' orientational distribution and the nematic order parameter $S$ were inferred from the ultrasonic measurements. The investigation of SAXS patterns reveals the orientation and sizes of aggregated structures under application of different magnetic-field strengths. In addition, the magnetic-field-dependent yield stress was measured, and a relationship between the yield stress and magnetic-field strength up to $0.5 \mathrm{~T}$ was established.
\end{abstract}

\section{Introduction}

Magnetic fluids (ferrofluids) are colloidal suspensions containing magnetic nanoparticles dispersed in an organic or inorganic liquid carrier. Due to their small core diameters, $d$, of $4-200 \mathrm{~nm}$, the particles generally form a single domain of uniform magnetization with a magnetic moment $\mu$ given by $\mu=M_{b} V$, where $M_{b}$ denotes the bulk magnetization of the particle material and $V=\pi d^{3} / 6$ is the volume of the core of the particle. Many applications of magnetic fluids require structural stability, i.e. no formation of aggregates even in strong magnetic fields. The aggregation of nanoparticles can be avoided by covering particles with a single or double layer of surfactant. However, large values of the coupling constant, $\lambda=\mu_{0} \mu^{2} / 4 \pi d_{h}{ }^{3} k_{B} T$ [1], and volume fractions of magnetic grains $\phi$ lead to the formation of magnetic aggregates in the form of chain-like structures. In the expression for $\lambda, d_{h}$ is the hydrodynamic diameter of the particle which is greater than the size of the magnetic core, $d$, by twice the thickness of protective surfactant layer [2]. Detecting the particle aggregation is

\footnotetext{
a e-mail: zbigniew.rozynek@ntnu.no
}

possible only by indirect experimental methods based on magnetorelaxometry [3], viscosity [4,5], ultrasound attenuation measurements [6], or SANS/SAXS [7-9] performed in external magnetic field.

Ferrofluids exhibit special properties that make them suited for many technical and medical applications. Some of these properties are determined by the particular physics of small-sized magnetic nanoparticles. Therefore it is important to search for better methods and theories describing the physical behaviour of these liquids, and in particular, the magnetic-field-induced structuring from magnetic particles. Concerning the measurements of the ferrofluid degree of anisotropy, typically scattering methods such as small-angle X-ray/neutron scattering (SAXS/SANS) are used and several approaches for the calculation of the nematic order parameter are common $[10,11]$. It can be seen that the value of the order parameter changes drastically when different methods of calculations are chosen. If for example the socalled "wide angle diffuse ring" approach is used (suggested by Leadbetter et al. [12]), several assumptions need to be applied and those normally tend to overestimate the value of the order parameter. The derivation of the 
orientational distribution functions from scattering data is generally complicated by the coupling between the orientational and positional orders of the scattering moieties. Therefore it is suggested here to employ a different measurement technique, i.e. ultrasonic spectroscopy and the theory described by Shliomis, Mond and Morozov [13] as an alternative approach to scattering methods.

The layout of this work is as follows. The sample preparation is first described briefly, followed by the first major part that focuses on the sample characterization. This includes determination of a particle average diameter, volume concentration and estimation of a coupling constant employing VSM (see below), rheology and SAXS. The second part covers the investigation of the $B$-field-induced structuring from magnetic particles. The average dimensions of the aggregated structures, their preferred orientation and nematic order parameter are studied with SAXS and ultrasonic spectroscopy measurements.

\section{Sample preparation}

The magnetic liquids composed of mineral oil ITO 100 and $\mathrm{Fe}_{3} \mathrm{O}_{4}$ particles coated with oleic acid were prepared in the Institute of Experimental Physics in Košice. Magnetic particles were obtained by chemical precipitation of ferrous and ferric salts in alkali medium. The appropriate amounts $\mathrm{FeSO}_{4}$ and $\mathrm{FeCl}_{3}$ solution were mixed and precipitated by a $25 \%(\mathrm{v} / \mathrm{v})$ solution of $\mathrm{NH}_{4} \mathrm{OH}$. Magnetite was formed as a black precipitate. To remove impurities formed during chemical reaction, the obtained magnetite particles were washed several times by magnetic decantation. After the last washing, the oleic acid (as surfactant to prevent irreversible aggregation of magnetic particles) and mineral oil ITO 100 (as carrier liquid) were added at $70^{\circ} \mathrm{C}$ in the volume ratio $1: 1$. Subsequently ITO 100 was added dropwise during stirring and heating in order to obtain the appropriate volume concentration of magnetite nanoparticles [14].

Samples with four different nanoparticle concentrations were prepared. The initial ferrofluid FF was diluted with mineral oil in the following proportions: FF:4, FF:64 and FF:128, respectively. The exact concentration value for the initial ferrofluid $\mathrm{FF}$ was obtained by means of the VSM method (see sect. 3.1).

\section{Experimental techniques and results}

\subsection{VSM-vibrating sample magnetometry}

A vibrating sample magnetometer (VSM) measures the magnetic hysteresis loop of the magnetic fluid studied. Volume concentrations of magnetite particles in the magnetic liquids, the average diameter of magnetite particles and the corresponding standard deviation were determined by VSM measurements.

The resulting magnetization curve is shown in fig. 1a. The magnetization of the diluted magnetic fluid can be
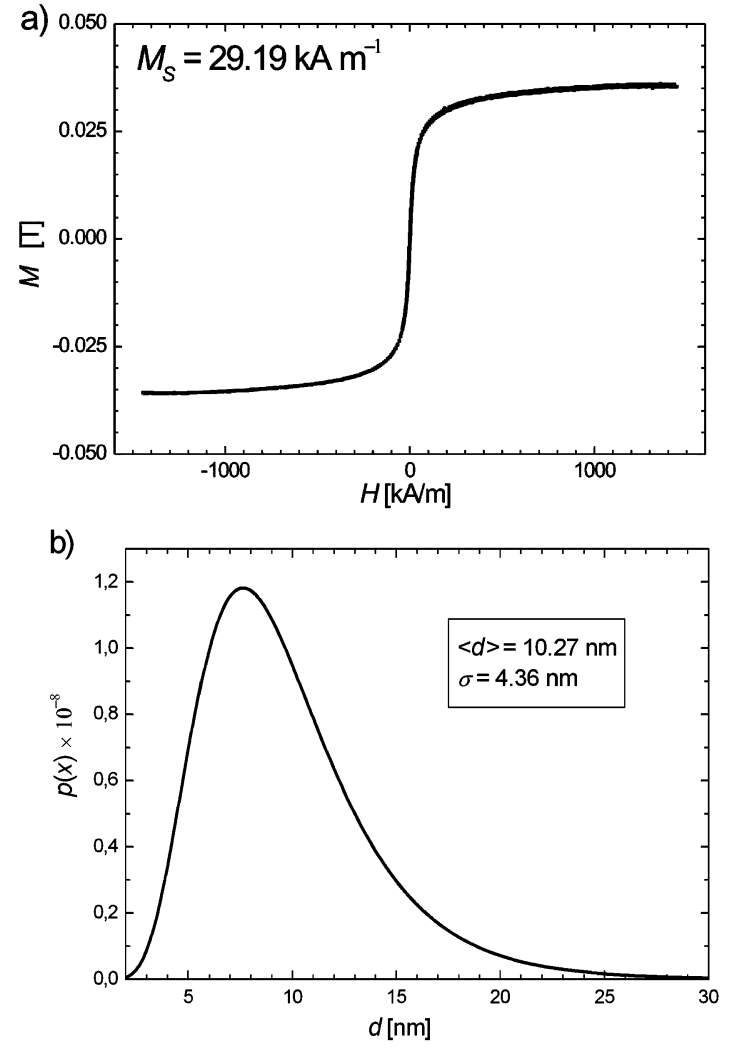

Fig. 1. Magnetization curve (a) and the particle size distribution function (b) for the mineral oil-based magnetic fluid obtained by means of the VSM method.

expressed as a superposition of the Langevin functions [2]

$$
M=M_{s} \int_{0}^{\infty} L(\xi) p(x) \mathrm{d} x
$$

where $M_{S}$ is the saturation magnetization, $L(\xi)=$ $\operatorname{coth}(\xi)-1 / \xi$ is the Langevin function with $\xi=$ $\mu_{0} \mu H / k_{B} T, \mu_{0}$ is the magnetic permeability of vacuum, $H$ is the magnetic-field strength, $k_{B}$ is the Boltzmann constant and $T$ is the absolute temperature. For the description of the dispersion of the magnetite particle size, the lognormal distribution is usually employed [2]

$$
p(x)=\frac{1}{x s \sqrt{2 \pi}} \exp \left(-\frac{\ln ^{2}(x / D)}{2 s^{2}}\right),
$$

where $D$ and $s$ are the parameters determined from the magnetization curve. On the basis of these parameters the mean diameter, $\langle d\rangle$, and standard deviation of particle size $\sigma$, can be determined from the formulae:

$$
\begin{aligned}
\langle d\rangle & =D \exp \left(\frac{s^{2}}{2}\right), \\
\sigma & =D \exp \left(\frac{s^{2}}{2}\right) \sqrt{\exp s^{2}-1} .
\end{aligned}
$$

The particle size distribution function $p(x)$ extracted from VSM measurements using eq. (1) with the parameter values of $M_{S}=29.19 \mathrm{kA} / \mathrm{m}, D=9.48 \mathrm{~nm}$, and $s=0.407$ is 
shown in fig. 1b. From the saturation magnetization $M_{S}$ the volume fraction of the magnetic phase can be determined via the expression

$$
\phi=\frac{M_{s}}{M_{b}},
$$

where $M_{b}=446 \mathrm{kA} / \mathrm{m}$ is the spontaneous magnetization of magnetite grains. In the case of the initial ferrofluid (FF) studied here, eq. (5) leads to a value $\phi=6.6 \%(\mathrm{v} / \mathrm{v})$. The particle size average value, $\langle d\rangle=10.27 \mathrm{~nm}$, obtained from VSM data using eq. (3), and hydrodynamic diameter $d_{h}=14.27 \mathrm{~nm}$, calculated on the assumption that the length of the oleic acid molecule equals $2 \mathrm{~nm}$, leads to a coupling constant $\lambda=0.54$. In conventional ferrofluids like maghemite $\left(\gamma-\mathrm{Fe}_{2} \mathrm{O}_{3}\right)$ or magnetite $\left(\mathrm{Fe}_{3} \mathrm{O}_{4}\right)$, with typical particle size $5-10 \mathrm{~nm}$, the magnetic interaction is generally weak $(\lambda<1)$, mainly reinforcing the vdW attraction [15]. Although the coupling constant $\lambda=0.54$ is relatively small and the studied fluid magnetic dipole interaction is rather weak, it is still possible for nanoparticles to form chain-like structures. This was confirmed by the rheological studies described in the next section and the chain-like structuring observed via SAXS investigation reported in the next paragraphs.

From the VSM data, the mean-spherical and the hightemperature approximation models give the same lognormal distribution of magnetite particle diameters $[16,17]$.

\subsection{Rheology}

\subsubsection{Experimental method}

The magneto-rheology (MR) of the ferrofluid was measured under different magnetic fields using an Anton Paar Physica MCR 300 rotational rheometer equipped with a thermostat unit MRD 180 and measuring system PP20/MR, specially designed for plate-plate-type MR measurements. All rheological measurements were undertaken at constant temperature $\left(21^{\circ} \mathrm{C}\right)$ and a gap of $0.2 \mathrm{~mm}$. The shear stress $\tau$ as a function of a shear rate $\gamma$ was measured by means of controlled shear rate (CSR) tests. For the samples in this study, and under application of a magnetic field, this dependence is well described by the Herschel-Bulkley rheological model: $\tau=\tau_{y}+b \dot{\gamma}^{p}$, where $\tau_{y}, b, p$ are constants named yield stress, consistency index, and power law index, respectively. The effective viscosity of the Herschel-Bulkley fluids upon deformation is not constant but follows a power law type behaviour in contrast to the constant viscosity found in Bingham fluids $[18,19]$.

\subsubsection{Rheology results}

In general, the yield stress values for ferrofluids are low in comparison with those of magnetorheological fluids constituting bigger particle (sizes in the range of $\mu \mathrm{m}$ ), since the maximum dipolar attraction (if two dipoles in head-to-tail

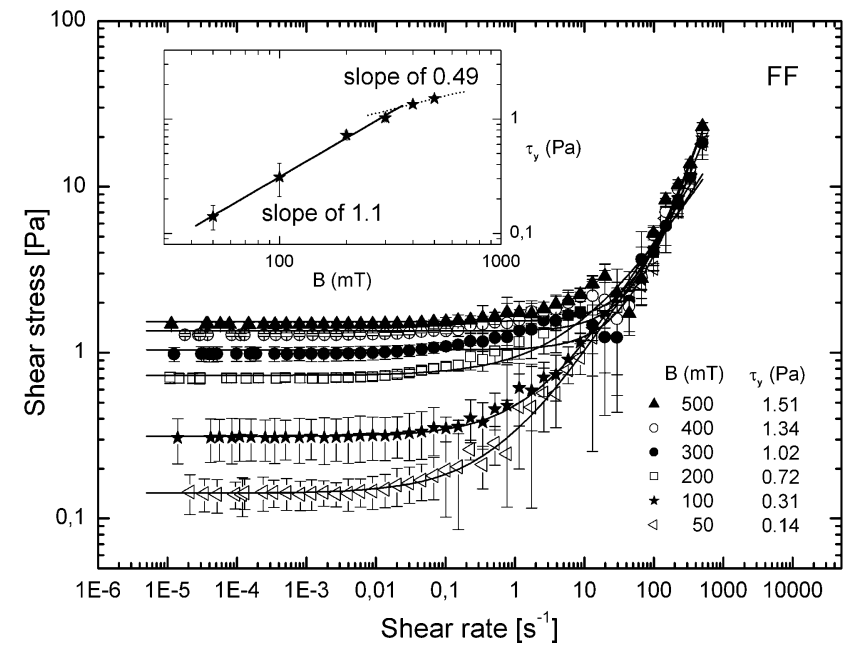

Fig. 2. A log-log plot of the flow curves of the initial ferrofluid (FF). The inset shows the yield stress $\tau_{y}$ dependence on the applied magnetic-field strength.

configuration are considered) scales as $V_{m} \propto d^{3}$, where $d$ is the core diameter, including the surfactant shell if it is present [20]. As suggested by Zubarev and Iskakova [21], the saturated magnitude of the yield stress (considering thin-film ferrofluids with thickness significantly smaller than $1 \mathrm{~mm}$ ) can be estimated using the equation

$$
\tau_{y}^{\mathrm{sat}}=0.162 \mu_{0} M_{b}^{2} \psi \cdot\left[\frac{\phi_{h}}{\left(1+2 d_{s} / d\right)^{3}}\right]^{2},
$$

where $\phi_{h}$ is the hydrodynamical concentration, $\psi$ is the concentration of particles that are big enough $(>18 \mathrm{~nm})$ and able to form large domains, $d_{s}$ is the thickness of the surface shell on the particle, and finally $d$ is the diameter of the magnetic core of the particle. When employing the following values: $d_{s}=2 \mathrm{~nm}, \phi_{h}=0.5$ (both assumed as typical values), $d=10.27 \mathrm{~nm}$ and $\psi$ varying from 0.001 to 0.002 (both provided by the VSM measurement, see fig. 1b), the calculated value of $\tau_{y}^{\text {sat }}$, based on eq. (6), lies between 1.4 and $2.8 \mathrm{~Pa}$, which encompasses the results presented below.

Figure 2 shows a log-log plot of the flow curves for our samples, fitted with the Herschel-Bulkley model. The yield stress values obtained from CSR measurements by fitting with this model are $0.14,0.31,0.72,1.02,1.34$ and $1.51 \mathrm{~Pa}$ for the magnetic-field strengths of 50, 100, 200, 300,400 and $500 \mathrm{mT}$, respectively. The dependence of the yield stress $\tau_{y}$ on the applied magnetic-field strength is presented in the log-log plot in the inset of fig. 2. From the fit (black solid line) a nearly linear power law was derived for the dependence on the magnetic-field strength $(B<300 \mathrm{mT})$ and the yield stress $\tau_{y}$ scales as $\tau_{y} \propto B^{\kappa}$, with the power law exponent $\kappa=1.1$. However, for higher values of the magnetic-field strength the power law exponent $\kappa$ decreases sharply below 1 (dotted line). Further increase of the magnetic field would make the saturation yield stress appear. 


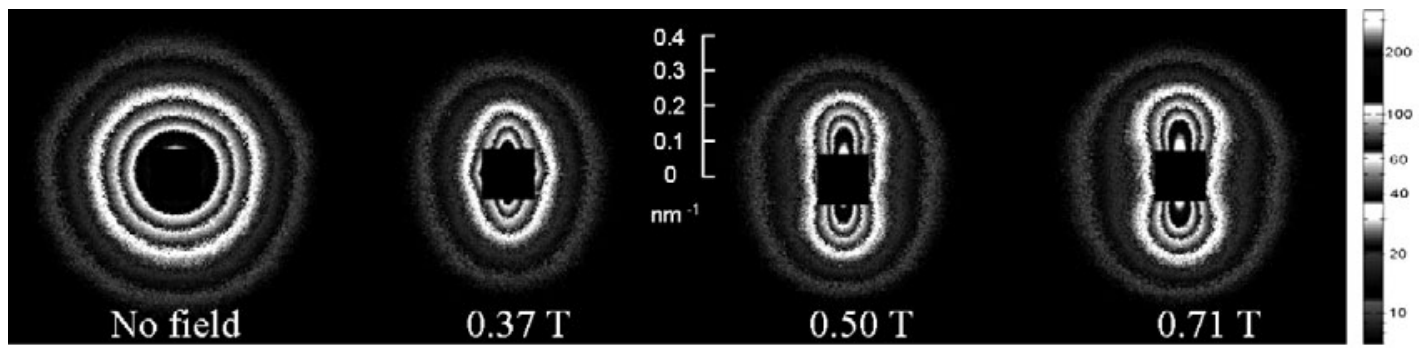

Fig. 3. Scattered intensity from FF:128 without and with horizontally applied magnetic field. Images were taken at the $q$-range corresponding to the distance $2 \pi / q$ of around $9-90 \mathrm{~nm}$.

\subsection{Small-angle X-ray scattering}

\subsubsection{Experimental method}

Small-angle X-ray scattering is a useful technique to study shape and size of macromolecules, characteristic distances of partially ordered materials, disordered structures and porous media. In a SAXS experiment, the dependence of the scattered intensity on the scattering angle is controlled by the size of the colloidal particles, their tendency to aggregate, the concentration of the dispersed system, the magnitude of the specific surface area, and more generally, by the inhomogenieties characterizing the structure of the disperse system. The scattered intensity $I(q)$, as a function of the momentum scattering vector $q$, given as $q=4 \pi \sin (\theta / 2) / \lambda$, where $\lambda$ is the wavelength of the incident beam and $\theta$ is the scattering angle, is proportional to the Fourier transform of the geometric correlation function of the electron density. The scattering vector $q$ has the dimension of the inverse length (i.e. $\AA^{-1}$ ), so that the length in real space corresponding to a certain $q$-value equals $2 \pi / q[22,23]$. The $\mathrm{X}$-ray scattering experiments were performed employing synchrotron radiation (ESRF, France). The experiment was conducted at the Dutch-Belgian Beamline (DUBBLE) at ESRF [24]. In order to induce alignment of the ferrofluid, different magnetic-field strengths, 0 to $0.71 \mathrm{~T}$, were employed. The beam was pointed along the radial direction of a $1 \mathrm{~mm}$ capillary whose cylindrical axis was vertical, and the magnetic field was applied horizontally, perpendicular to the X-ray beam. An X-ray beam with a wavelength of $1.033 \AA$ was used. The sample-to-detector distance was calibrated and set to $7 \mathrm{~m}$, enabling the scattering detection in a $q$-range of approximately $0.007-0.07 \AA^{-1}$. For the SAXS measurements in a magnetic field, samples were prepared in capillaries and placed between the poles of a permanent magnet. The gap of the latter can be continuously varied between 20 and $3 \mathrm{~mm}$, which allowed changing the field strength from 0.37 to $0.71 \mathrm{~T}$.

\subsubsection{SAXS results}

When the dipolar interactions are much stronger than the thermal energies (i.e. $\lambda \gg 1$ or when an external magnetic field is applied), the fluid develops clusters (dimers, trimers, etc.) and longer chains. Particles of arbitrary shape would exhibit statistically isotropic scatter-

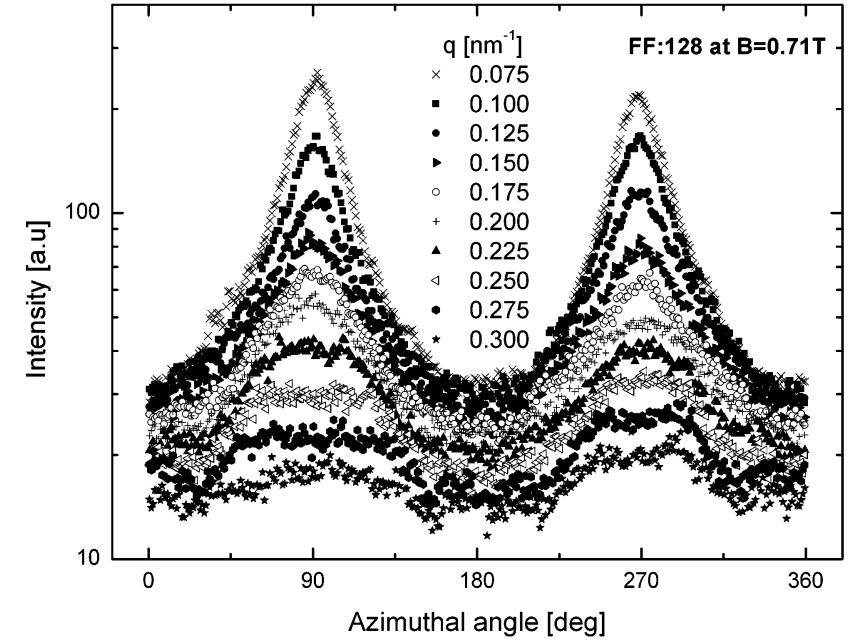

Fig. 4. Azimuthal plots of SAXS intensities at different $q$-ranges.

ing if they are randomly oriented, i.e., the measured intensity depends only on the magnitude of the scattering vector $q$, whereas oriented anisometric particles result in anisotropic scattering patterns [25].

Figure 3 shows the 2D SAXS patterns with and without a horizontally applied external magnetic field. For $B=0$ the intensity pattern is isotropic and it does not depend on the direction of the scattering vector $q$. However, when the magnetic field is applied, anisotropy develops and the scattering intensity is both radially and azimuthally dependent. In particular, the intensity perpendicular to, the field direction increases, while it decreases in the parallel direction. Since the particles have a diameter starting at about $5 \mathrm{~nm}$ (see fig. 1b), or ca. $9 \mathrm{~nm}$ when including the surfactant layer, the observable anisotropy caused by the smallest aggregated structure, namely a dimer, can be seen for the $q$-range starting from around $0.35 \mathrm{~nm}^{-1}$ and lower, which corresponds to $2 \pi / q>18 \mathrm{~nm}$ in real space. This is observed in fig. 3 , where the application of an external field changes the pattern from being circular at no field to elliptical when the field is present. The anisotropy is more pronounced when approaching the lower $q$-range (down to $q=0.09 \mathrm{~nm}^{-1}$ for sample FF: 128 at $B=0.71 \mathrm{~T})$. This is clearly shown in the azimuthal plots in fig. 4. Interestingly, the anisotropy reaches a maximum at a certain $q$-value $\left(q_{\text {an }}\right)$ and then the isotropy 

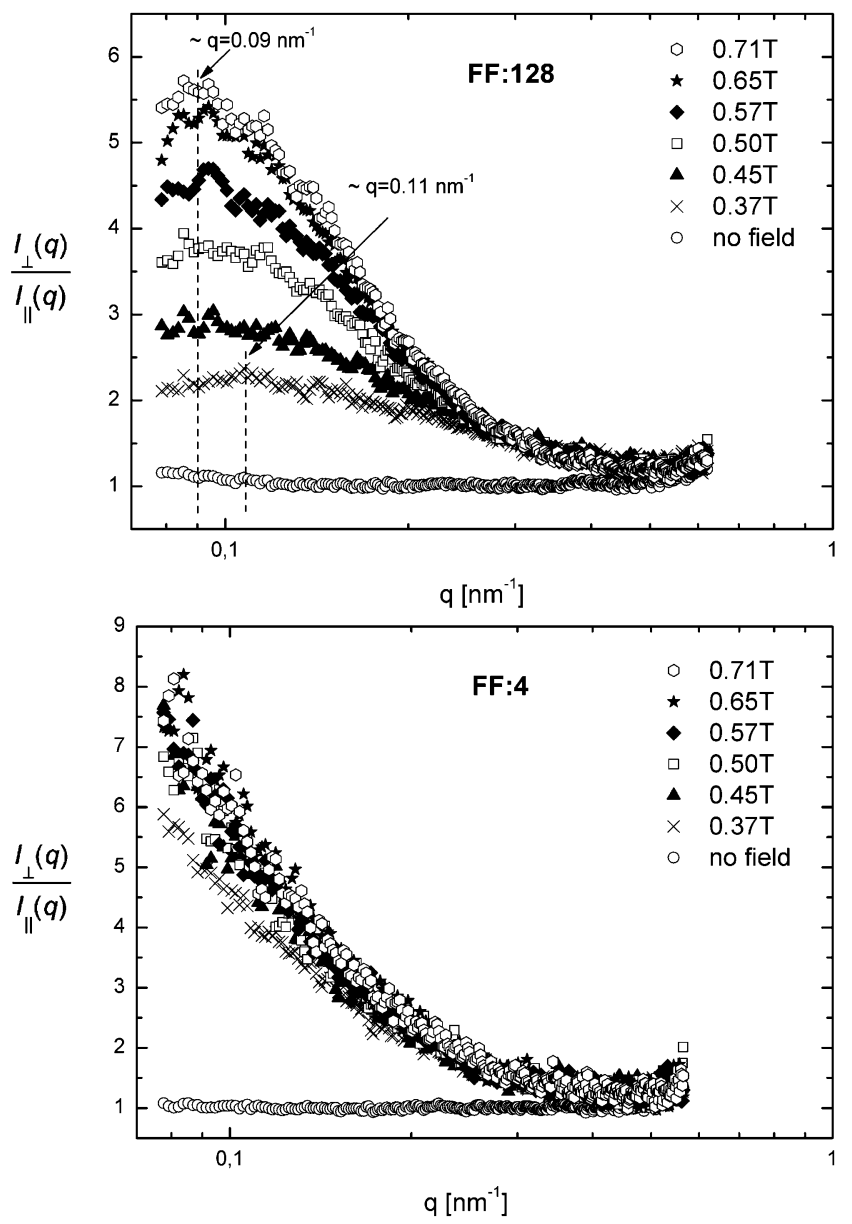

Fig. 5. Ratio of intensities normal and parallel to the magnetic-field direction as a function of wave vector $q$ for samples FF:128 (top) and FF:4 (bottom), respectively.

starts to recover leaving a long and $H / k T$-dependent tail. This, however, is not readily observed form either fig. 3 or fig. 4 , but can be revealed by plotting the ratio of the intensities $I_{\perp}(q) / I_{\|}(q)$ (perpendicular and parallel to the $B$-field direction) as a function of the wave vector $q$ shown in fig. 5 (top). The ratio displays maxima shifting from $q_{\text {an }} \sim 0.11 \mathrm{~nm}^{-1}$ to $q_{\text {an }} \sim 0.09 \mathrm{~nm}^{-1}$ for sample FF: 128 at magnetic-field strengths of $0.37 \mathrm{~T}$ and $0.71 \mathrm{~T}$, respectively. The peak's broadening decreases when the magnetic field strength increases indicating gradually more well-defined ordering. In the thermodynamic limit at the lowest values of $q$ the scattering approaches an isotropic behaviour, while at the highest $q$ isotropy is recovered because we have reached the size of the primary particles in the aggregates, which are spherical. Such behavior suggests that the aggregates are non-spherical assemblies of primary particles that become oriented in the magnetic field: as $B$ increases the radius of gyration (of the aggregated structure) in the parallel direction increases, while that in the perpendicular direction decreases (or remains the same) [26]. If the position of the peak centers (marked with dashed lines) is used as an estimate of the aggregate radius of gyration (as proposed by Teixeira et al. [26]), i.e.

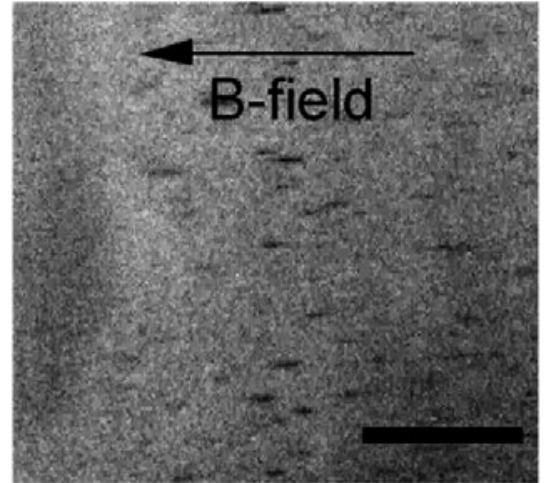

Fig. 6. Optical microscope image of FF:4 at approximately $0.5 \mathrm{~T}$. The length of the bar corresponds to $10 \mu \mathrm{m}$.

$R_{g} \sim 2 / q_{\text {an }}$, one obtains overall aggregate sizes $2 R_{g}$ from about 115 to $140 \mathrm{~nm}$ for FF:128, depending on the field strength. There is however no peak present in the probed $q$-range when the intensity ratio is plotted for a sample with high particle concentration (FF:4), see fig. 5 (bottom). This indicates that larger structures are formed for samples with high particle concentration (above 1\%) in comparison with those formed in diluted samples. In addition, it can be seen that a significant anisotropy starts building up already at a lower value of the $B$-field. The influence of polydispersity on the structural behaviour cannot be neglected. In particular for highly concentrated samples the formation of both chain-like structures with higher-order oligomers and other forms of aggregates are expected. It can thus be assumed that in the low $q$-range direction (outside our SAXS $q$-range) the $I_{\perp}(q) / I_{\|}(q)$ ratio has a long tail reaching $q \sim 0.03 \mathrm{~nm}^{-1}$ or lower (see also ref. [26]). One may even expect some of the sub- $\mu \mathrm{m}$ clusters to appear in the presence of high magnetic field. This was in fact observed for the sample with high particle concentration (FF:4) using optical microscopy, as shown in fig. 6. Note that the size of the aggregates cannot be precisely retrieved, since the image was captured with a certain exposure time during particle motion and was also enhanced by adjusting contrast and brightness.

The derivation of the orientational distribution functions from scattering data is generally complicated by the coupling between the orientational and positional orders of the scattering moieties. In the field of liquid crystals, the nematic order parameter $S$ is usually calculated either by considering single-molecule scattering, so that positional correlations are irrelevant, or by assuming ideal positional correlations within a small group of molecules [27]. The situation in our system is not trivial, since there might be no positional correlations within the $q$-range probed. The azimuthal intensity variations (and thus the nematic order parameter $S$ that depends on the azimuthal peak width) are also $q$-dependent as shown in fig. 4 . In addition, if one assumes the formation of a rod-like structure, a further broadening of the azimuthal peak width is expected due to the rod diameter variations and the structure's finite size in general. Therefore only an analysis in qualitative terms can be presented here and relative changes between the 


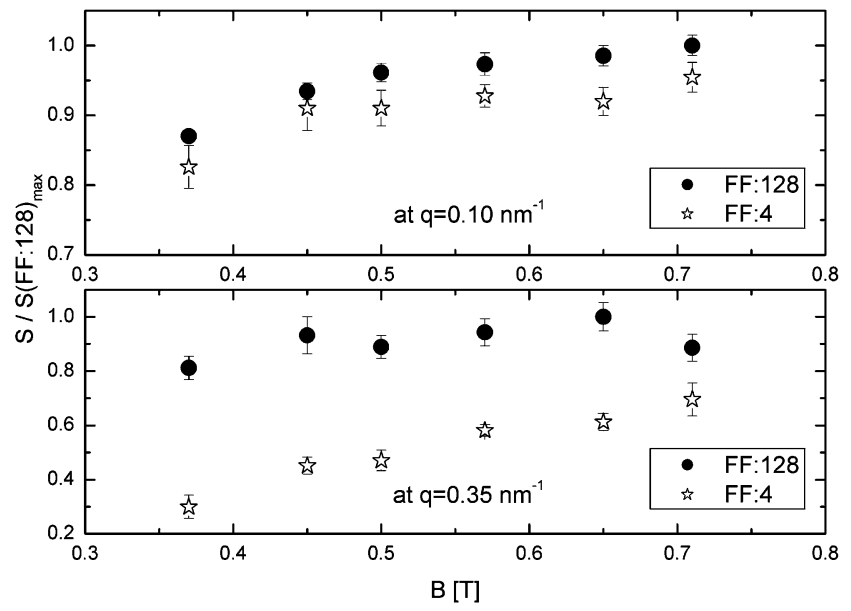

Fig. 7. Normalized order parameter for samples FF:4 (with high particle concentration) and FF:128 (diluted sample) calculated at different $q$-ranges.

order parameter values for samples FF:4 and FF:128 are illustrated in fig. 7. The order parameter was calculated according to the so-called "wide-angle diffuse ring" approach, where azimuthal plots (examples shown in fig. 4) were fitted using the classical Maier-Saupe distribution function $g(\beta)$, and the order parameter $S$ is given as

$$
\begin{aligned}
g(\beta) & \sim \exp \left(m \cos ^{2} \beta\right), \\
S_{n} & =\frac{1}{2}\left\langle 3 \cos ^{2} \beta-1\right\rangle_{g},
\end{aligned}
$$

where $m$ is a parameter that controls the width of the distribution and $\beta$ is the angle between the major chain axis and the nematic director (along the $B$-field). Along the whole detectable $q$-range, the order parameter values for the sample with high particle concentration $\mathrm{FF}: 4$ were found to be lower than those from the diluted sample FF:128. The order parameter values shown in fig. 7 for FF:4 and FF128 at $q=0.1$ and $q=0.35 \mathrm{~nm}^{-1}$ (top and bottom panels, respectively) have been normalized to the maximum value of $S$ for FF:128. Normally the thermal motion as well as the interaction between the magnetic moments of the particles leading to more complex structure formation (such as rings, clusters, etc.) will prevent a perfect alignment. The latter effect is more pronounced for samples with higher particle concentration and it can explain a lower degree of anisotropy for FF:4 in comparison to FF:128.

\subsection{Ultrasonic spectroscopy}

\subsubsection{Experimental method}

In the ultrasonic measurements a broad-band transducer (Optel) with centre frequency of $5 \mathrm{MHz}$ was used. The power spectrum of the echo signal was determined by using a Fast Fourier Transform algorithm. The measuring cell (made of brass) with fixed distance between transducer and reflector was used. The path length (back and forth) traversed by the ultrasonic pulse inside the medium was $12 \mathrm{~mm}$. The attenuation coefficient measured in excess to that of reference medium (water) can be expressed as

$$
\alpha(f)=-\frac{1}{2 L} \ln \frac{P_{\mathrm{m}}(f)}{P_{\mathrm{w}}(f)}+\frac{1}{2 L} \ln \frac{R_{\mathrm{mb}}}{R_{\mathrm{wb}}},
$$

where $P_{\mathrm{m}}(f), P_{\mathrm{w}}(f)$ are the power spectrums of the echo signal reflected from the wall of the measuring cell filled with magnetic liquid and water, respectively, $R_{\mathrm{mb}}=$ 0.9319 is the acoustic power reflection coefficient at the inner side of the measuring cell containing magnetic liquid, and $R_{\mathrm{wb}}=0.8314$ is the acoustic power reflection coefficient at the inner side of the measuring cell containing water. The accuracy of the ultrasonic measurements described above amounted to about $\pm 2-5 \%$. For the angulardependence experiment the magnetic field was rotated by ten degrees each time, while the measuring cell remained stationary in the gap between pole pieces of an electromagnet which produced a field of $75 \mathrm{mT}$. The magneticfield strength was measured with a Resonance Technology RX21-type teslameter to within $0.5 \%$.

\subsubsection{Ultrasonic anisotropy-results}

The compression or stretching of the chain-like aggregates under the influence of the ultrasonic wave results in a magnetic restoring force which leads to forced oscillations of the aggregates. This mechanism was first proposed by Taketeomi [28] and later reconsidered by Pleiner and Brand [29] and Shliomis, Mond and Morozov [13]. The structural changes are manifested by a dependence of the ultrasonic wave absorption on the direction of the wave with respect to the magnetic field. According to Shliomis, Mond and Morozov [13], two-particles chains (dimers) oscillations in the ultrasonic field lead to additional dissipation of ultrasonic wave energy with the coefficient of absorption given by

$$
\alpha_{\mathrm{dim}}=\frac{\eta \phi_{d}}{2 \rho c^{3}} \frac{\left\langle r^{2}\right\rangle}{d^{2}} \frac{\omega^{4} \tau^{2}}{1+\omega^{2} \tau^{2}} F(\varphi)
$$

where $c$ is the velocity of the ultrasonic wave propagating with angular frequency $\omega, \tau$ is the relaxation time of the dimer oscillations scaled by the Brownian diffusion time $\tau_{B}=3 \eta V / k_{B} T$ for a single particle $\left(\tau \approx \tau_{B}\right), \rho$ is the mass density and $\eta$ the shear viscosity of the magnetic liquid, respectively, $\phi_{d}$ is the volume fraction the dimers, and $\langle r\rangle$ is the average distance between magnetic particles in the dimer. The anisotropy function $F(\varphi)$ can be expressed by a field-induced nematic order parameter $S(\lambda, \xi)=1 / 2\left(3\left\langle\cos ^{2} \beta\right\rangle-1\right), \beta$ being the angle between the dimer axis and the applied magnetic field $H$ (similarly as eq. (8)), through the relation

$$
F(\varphi)=\left[1+S\left(3 \cos ^{2} \varphi-1\right)\right]^{2},
$$

where $\varphi$ is the angle between the wave vector and the direction of the external magnetic field. The upper graph in 

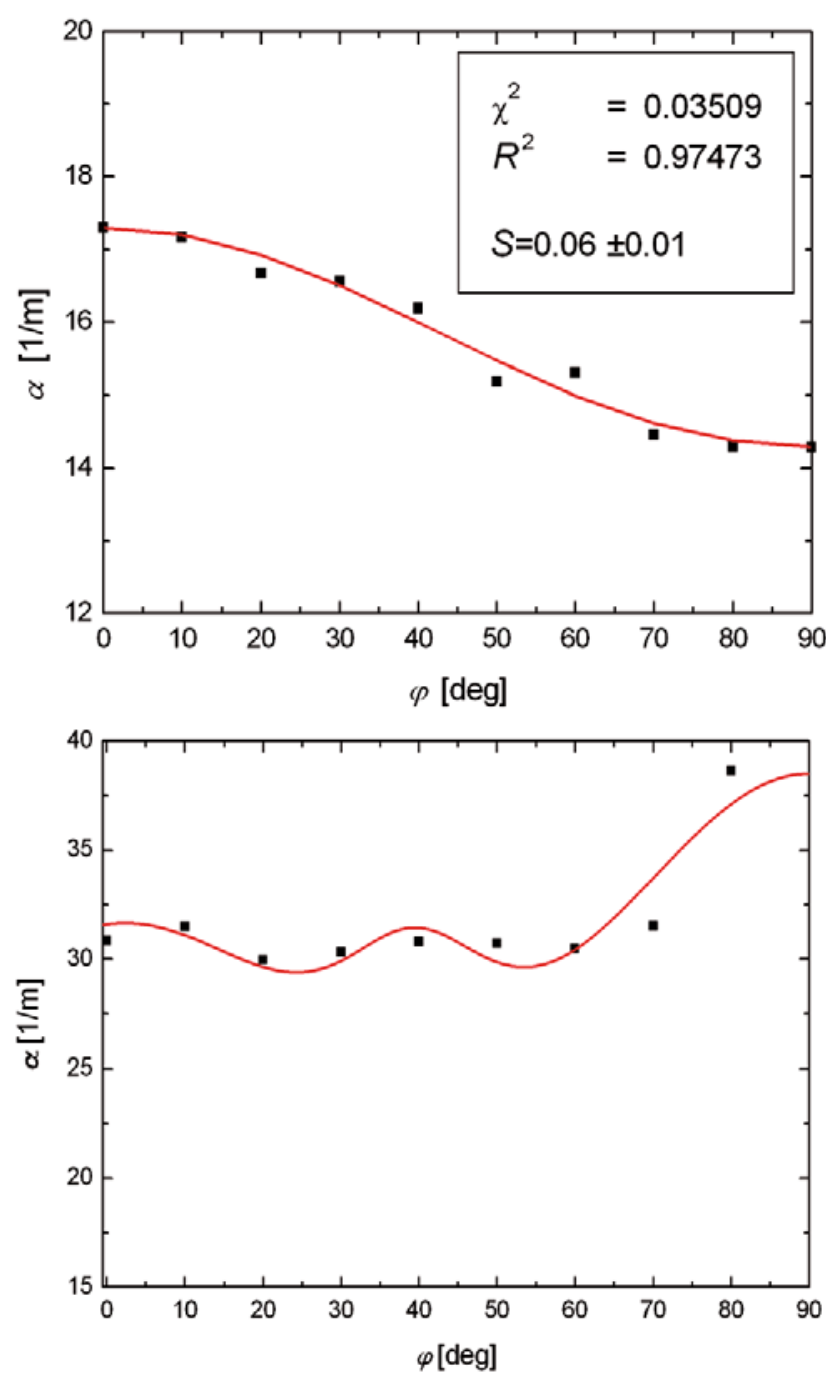

Fig. 8. Angular dependence of the ultrasonic attenuation $\alpha(\varphi)$ for a wave of frequency $f=5 \mathrm{MHz}$ in mineral oil-based magnetic liquid in an external magnetic field of $B=75 \mathrm{mT}$. Upper graph shows the results for a diluted magnetic fluid (FF:64) and lower graph for a concentrated fluid (FF:4). The solid line on the upper graph was calculated using eq. (12).

fig. 8 shows the results of the ultrasonic anisotropy data $\alpha_{\operatorname{dim}}(\varphi)$ for the diluted mineral oil-based magnetic liquid with concentration (FF:64) subjected to a constant magnetic field of $B=75 \mathrm{mT}$. The solid line was obtained by fitting the expression

$$
\frac{\alpha(\varphi)-\alpha(\pi / 2)}{\alpha(0)-\alpha(\pi / 2)}=\frac{2(1-S) \cos ^{2} \varphi+3 S \cos ^{4} \varphi}{2+S},
$$

following from the Shliomis, Mond and Morozov [13] theory to the experimental data. The observed dependence of $\alpha_{\mathrm{dim}}$ on $\varphi$ is characteristic of short-chains aggregates that are formed as a result of the pair interparticle magnetic dipole-dipole interaction. More specifically, there is a monotonic decrease of the ultrasonic attenuation with increasing $\varphi$, and the attenuation is maximal when the
Table 1. Characteristic particle features - summary.

\begin{tabular}{lcc}
\hline Volume fraction $\phi$ & 6.6 & $(\%)$ \\
Mean diameter $\langle d\rangle$ & 10.27 & $(\mathrm{~nm})$ \\
Hydrodynamic diameter $d_{h}$ & 14.27 & $(\mathrm{~nm})$ \\
Coupling constant $\lambda$ & 0.54 & \\
Saturated yield stress $\tau_{y}^{\text {sat }}$ & $1.4-2.8$ & $(\mathrm{~Pa})$ \\
Order parameter $S$ & 0.06 & \\
\hline
\end{tabular}

field is applied parallel to the direction of the sound propagation and minimal when the field is applied perpendicularly. It should be noted that this behaviour is characteristic only to the weakly concentrated magnetic fluids with predominant presence of two-particle chains. In a more concentrated magnetic fluids (FF:4), lower graph in fig. 8, the structure of the aggregates and angle dependence of the ultrasound coefficient become much more complicated and cannot be explained using the mechanism suggested in the Shliomis, Mond and Morozov theory [30]. This conclusion is supported by the analysis of the SAXS data already discussed in the previous section. The value of the nematic order parameter $S=0.06 \pm 0.01$ calculated from the best fit of eq. (10) to ultrasonic data is in agreement with the theoretical value of $S$ calculated for $\lambda=0.54$ and $\xi=5.43[13]$.

\section{Conclusions}

This study has been focused on the magnetic-fieldinduced structuring from nanoparticles. A considerable part also covers particle characterization without field, since it is important to know in advance the particle average size $d$ and the size distribution $\sigma$ in order to estimate the coupling constant $\lambda$ and predict the particle behavior in the colloidal system. Those initial parameters were obtained using the VSM method, giving an average particle diameter $d=10.27 \mathrm{~nm}$ and a hydrodynamic diameter $d_{h}=14.27 \mathrm{~nm}$. In table 1 the characteristic parameters of the system studied are summarized. The list includes the volume fraction $\phi$, the average particle diameter, the value of the coupling constant $\lambda$, the yield stress, and finally the order parameter acquired from ultrasonic spectroscopy.

The nematic order parameter found by means of ultrasonic spectroscopy converges well with the theoretical values only for low concentrated magnetic fluids. This is due to the structure-size distribution independence of the Shliomis, Mond and Morozov method, since it is assumed that the main building block of the system is a dimer, and larger structures ( $N$-particle oligomers) may be considered as short flexible chains (with $3 N-6$ oscillatory degrees of freedom) represented also as a single dimer (see ref. [13]).

The analysis of the two-dimensional SAXS showed a development of the anisotropy starting from a $q$-range of around $0.35 \mathrm{~nm}^{-1}$ (corresponding to the size of a dimer). 
The anisotropy was more pronounced when approaching a lower $q$-range (down to $q_{\mathrm{an}}=0.09 \mathrm{~nm}^{-1}$ for sample FF:128 at $B=0.71 \mathrm{~T}$ ) and interestingly, the anisotropy reached its maximum value and peaks at $q_{\text {an }}$ and then the isotropy started to recover leaving a long and $H / k T$-dependent tail. This was revealed by plotting the ratio of the intensities $I_{\perp}(q) / I_{\|}(q)$ (perpendicular and parallel to the $B$-field direction) as a function of the wave vector $q$ shown in fig. 5 (top). The ratio displayed maxima shifting from $q_{\mathrm{an}}^{0.35 T} \sim$ $0.11 \mathrm{~nm}^{-1}$ to $q_{\mathrm{an}}^{0.71 T} \sim 0.09 \mathrm{~nm}^{-1}$ for sample $\mathrm{FF}: 128$ at magnetic-field strengths of $0.37 \mathrm{~T}$ and $0.71 \mathrm{~T}$, respectively. The aggregate radius of gyration changed from 115 to $140 \mathrm{~nm}$ for FF:128, depending on the field strength. There was however no peak present at the detectable $q$-range for a sample with high particle concentration (FF:4). This indicates that larger structures were formed for samples with high particle concentration (above 1\%) in comparison with those formed in diluted samples. The relative values of the nematic order parameter were calculated via fits to azimuthal plots of the SAXS data. It was found that along the whole detectable $q$-range, the relative order parameter values for the sample with high particle concentration FF:4 were lower than those from the diluted sample FF:128. The lower degree of anisotropy for FF:4 in comparison to FF:128 was caused by the formation of more complex structure formations (such as rings, clusters, etc.) that prevented a better alignment. This was also confirmed by the ultrasonic spectrometry.

In addition, the magnetic-field-dependent yield stress was measured, and a relationship between the yield stress and the magnetic-field strength up to $500 \mathrm{mT}$ was obtained. Based on the results from VSM, the value of the saturated magnitude of the yield stress $\tau_{y}^{\text {sat }}$ was estimated to be between 1.4 and $2.8 \mathrm{~Pa}$, which converged well with the results obtained from rheometry. These results provide once more the experimental confirmation of the recently described theory by Zubarev and Iskakova.

This work was supported by the Research Council of Norway through the FRINAT Program: NFR project number 171300. We are grateful for beam time at the Dutch-Belgian Beamline at the European Synchrotron Radiation Facility, Grenoble. The studies were supported by Slovak Academy of Sciences VEGA 2/0077/09, Nanofluid, Centre of Excellence, projects APVV 0509-07, MNT-ERA Net and SK-PL-0069-09/8158/2010. The authors acknowledge A.P. Hammersley, author of the Fit2D program.

Open Access This article is distributed under the terms of the Creative Commons Attribution Noncommercial License which permits any noncommercial use, distribution, and reproduction in any medium, provided the original author(s) and source are credited.

\section{References}

1. S. Odenbach, J. Phys.: Condens. Matter 16, 1135 (2004).

2. M. Raşa, Eur. Phys. J. 2, 265 (2000).

3. D. Eberbeck, F. Wiekhorst, U. Steinhoff, L. Trahms, J. Phys.: Condens. Matter 18, 2829 (2006).

4. S. Odenbach, K. Raj, Magnetohydrodynamics 36, 312 (2000).

5. H. Shahnazian, S. Odenbach, J. Phys.: Condens. Matter 20, 204137 (2008).

6. A. Józefczak, A. Skumiel, J. Phys.: Condens. Matter 18, 1869 (2006).

7. F.L.O. Paula, R. Aquino, G.J. da Silva, J. Depeyrot, F.A. Tourinho, J.O. Fossum, K.D. Knudsen, J. Appl. Cryst. 40, 269 (2007).

8. F.L.O. Paula, G.J. da Silva, J.O. Fossum, K.D. Knudsen, G. Helgesen, R. Aquino, F.A. Tourinho, J. Depeyrot, Braz. J. Phys. 39, 163 (2009).

9. H. Shahnazian, S. Odenbach, Phys. Rev. B 67, 094206 (2003).

10. P. Davidson, D. Petermann, A.M. Levelut, J. Phys. II 5, 113 (1995).

11. L.M. Pop, S. Odenbach, J. Phys.: Condens. Matter 18, 2785 (2006).

12. A.J. Leadbetter, E.K. Norris, Mol. Phys. 38, 669 (1979).

13. M. Shliomis, M. Mond, K. Morozov, Phys. Rev. Lett. 101, 074505 (2008).

14. F. Herchl, P. Kopčanský, M. Timko, M. Koneracká, K. Marton, I. Kolcunová, L. Tomčo, Acta Phys. Pol. A 113, 569 (2008).

15. C. Holm, J.J. Weis, Curr. Opin. Colloid Interface Sci. 10, $133(2005)$.

16. A. Skumiel, Int. J. Thermophys. 31, 546 (2010).

17. A. Skumiel, T. Hornowski, A. Józefczak, Int. J. Thermophys. DOI 10.1007/s10765-010-0799-4 (2011).

18. K.P. Vishwanath, A. Kandasamy, Appl. Math. Mod. 34, 219 (2010).

19. A.N. Alexandrou, T.M. McGilvreay, G. Burgos, J. NonNewtonian Fluid Mech. 100, 77 (2001).

20. M. Klokkenburg, B.H. Erné, A. Wiedenmann, A.V. Petukov, A.P. Philipse, Phys. Rev. E 75, 051408 (2007).

21. A.Y. Zubarev, L.Y. Iskakova, Physica A 365, 265 (2006).

22. O. Glatter, O. Kratky, Small-Angle X-ray Scattering (Academic, New York, 1982).

23. P. Linder, T. Zemb, Neutrons, X-rays and Light: Scattering Methods Applied to Soft Condensed Matter (North Holland, 2002).

24. W. Bras, I.P. Dolbnya, D. Detollenaere, R. van Tol, M. Malfois, G.N. Greaves, A.J. Ryan, E. Heeley, J. Appl. Cryst. 36, 791 (2003).

25. F. Dobrich, A. Michels, R. Birringer, J. Magn. \& Magn. Mater. 316, 779 (2007).

26. A.V. Teixeira, I. Morfin, F. Ehrburger-Dolle, C. Rochas, P. Panine, P. Licinio, E. Geissler, Compos. Sci. Technol. 63, 1105 (2003).

27. B.J. Lemaire, P. Panine, J.C.P. Gabriel, P. Davidson, Europhys. Lett. 59, 55 (2002).

28. S. Taketomi, J. Phys. Soc. Jpn. 55, 838 (1986).

29. H. Pleiner, H.R. Brand, J. Magn. \& Magn. Mater. 85, 125 (1990).

30. V.V. Sokolov, Acoust. Phys. 56, 972 (2010). 